\title{
Higher-order integrable evolution equation and its soliton solutions
}

\author{
Adrian Ankiewicz and Nail Akhmediev \\ Optical Sciences Group, Research School of Physics and Engineering, \\ The Australian National University, Canberra ACT 0200, Australia
}

\begin{abstract}
We consider an extended nonlinear Schrödinger equation with higher-order odd (third order) and even (fourth order) terms with variable coefficients. We demonstrate its integrability, provide its Lax pair, and, applying the Darboux transfromation, present its first and second order soliton solutions. The equation and its solutions have two free parameters. Setting one of these parameters to zero admits two limiting cases: the Hirota equation on the one hand and the Lakshmanan - Porsezian Daniel (LPD) equation on the other hand. When both parameters are zero, the limit is the nonlinear Schrödinger equation.
\end{abstract}

PACS numbers: PACS numbers: 05.45.Yv, 42.65.Tg, 42.81.Qb

In this letter, we consider the following evolution equation which includes higher-order even and odd terms:

$$
\begin{aligned}
i \psi_{x}+\frac{1}{2} \psi_{t t}+|\psi|^{2} \psi-i \alpha\left(\psi_{t t t}+6 \psi_{t}|\psi|^{2}\right) & \\
+\gamma\left(\psi_{t t t t}+6 \psi_{t}^{2} \psi^{*}+\right. & 4 \psi\left|\psi_{t}\right|^{2}+8 \psi_{t t}|\psi|^{2} \\
+ & \\
\left.+2 \psi_{t t}^{*} \psi^{2}+6 \psi|\psi|^{4}\right) & =0 .
\end{aligned}
$$

Here $x$ is the propagation variable and $t$ is time in the moving frame, with the function $\psi(x, t)$ being the envelope of the waves. The notation is standard in the theory of nonlinear waves. Sometimes $x$ and $t$ are interchanged in optics and water wave theory $[1,2]$. All coefficients in this equation are fixed except the two variables: $\alpha$ and $\gamma$. When both coefficients are zero, the remaining part is the standard normalised nonlinear Schrödinger equation [3]. If only $\gamma=0$, the equation is found to be integrable and is known as Hirota equation [4]. Furthermore, when only $\alpha=0$, the equation is also integrable and known as the 'Lakshmanan - Porsezian - Daniel' (LPD) equation [5]. However, the integrability of the combined equation, to the best of our knowledge, has not been considered so far. form:

The equation (1) can also be written in the operator

$$
\begin{aligned}
& F[\psi(x, t)]= \\
& S[\psi(x, t)]-i \alpha H[\psi(x, t)]+\gamma P[\psi(x, t)]=0,
\end{aligned}
$$

where $S$ is the nonlinear Schrödinger operator:

$$
S[\psi(x, t)]=i \psi_{x}+\frac{1}{2} \psi_{t t}+\psi|\psi|^{2},
$$

$H$ is the Hirota operator:

$$
H[\psi(x, t)]=6 \psi_{t}|\psi|^{2}+\psi_{t t t},
$$

and $P$ is the LPD operator:

$$
\begin{aligned}
P[\psi(x, t)] & =\psi_{t t t t}+6 \psi|\psi|^{4}+4 \psi\left|\psi_{t}^{*}\right|^{2} \\
& +8 \psi_{t t}|\psi|^{2}+6 \psi_{t}^{2} \psi^{*}+2 \psi^{2} \psi_{t t}^{*}
\end{aligned}
$$

We can present Eq.(1) in the form of the condition of compatibility of two linear equations:

$$
\begin{aligned}
\mathbf{R}_{t} & =\mathbf{L R}, \\
\mathbf{R}_{x} & =\mathbf{B R},
\end{aligned}
$$

where the vector

$$
\mathbf{R}=\left[\begin{array}{l}
r \\
s
\end{array}\right]
$$

consists of two complex functions $r=r(x, t)$ and $s=$ $s(x, t)$ while the matrix operators

$$
\mathbf{L}=\lambda \mathbf{J}+\mathbf{U}
$$

and

$\mathbf{B}=\lambda^{2} \mathbf{J}(\mathbf{x}, \mathbf{t})+\lambda \mathbf{U}(\mathbf{x}, \mathbf{t})+\frac{1}{2} \mathbf{V}(\mathbf{x}, \mathbf{t})-\alpha \mathbf{M}(\mathbf{x}, \mathbf{t})+\gamma \mathbf{V}_{\mathbf{p}}(\mathbf{x}, \mathbf{t})$

can be written in terms of the following square matrices:

$$
\mathbf{U}=\left[\begin{array}{cc}
0 & i \psi^{*}(x, t) \\
i \psi(x, t) & 0
\end{array}\right], \mathbf{J}=\left[\begin{array}{cc}
i & 0 \\
0 & -i
\end{array}\right]
$$

and

$$
\mathbf{V}=\left[\begin{array}{cc}
-i|\psi|^{2} & \psi_{t}^{*} \\
-\psi_{t} & i|\psi|^{2}
\end{array}\right]
$$

For arbitrary complex $\lambda$, we can define the operator $\mathrm{M}$ :

$$
\mathbf{M}=4 \lambda^{3} \mathbf{J}(\mathbf{x}, \mathbf{t})+4 \lambda^{2} \mathbf{U}(\mathbf{x}, \mathbf{t})+2 \lambda \mathbf{V}(\mathbf{x}, \mathbf{t})+\mathbf{K}_{\mathbf{a}}(\mathbf{x}, \mathbf{t})
$$

where

$$
\mathbf{K}_{\mathbf{a}}=\left[\begin{array}{cc}
A_{n}(x, t) & -B_{n}(x, t)^{*} \\
B_{n}(x, t) & -A_{n}(x, t)
\end{array}\right],
$$


with

$$
\begin{aligned}
& A_{n}(x, t)=\psi^{*} \psi_{t}-\psi \psi_{t}^{*} \\
& B_{n}(x, t)=-i\left(2 \psi|\psi|^{2}+\psi_{t t}\right) .
\end{aligned}
$$

In our notation, the last matrix, $\mathbf{V}_{\mathbf{p}}$, is:

$$
\mathbf{V}_{\mathbf{p}}=\left[\begin{array}{cc}
i A_{p}(x, t) & B_{p}(x, t) \\
-C_{p}(x, t) & -i A_{p}(x, t)
\end{array}\right]
$$

where

$$
\begin{array}{r}
A_{p}(x, t)=-3|\psi|^{4}+\left|\psi_{t}\right|^{2}-2 i \lambda\left(\psi \psi_{t}^{*}-\psi^{*} \psi_{t}\right) \\
-\psi^{*} \psi_{t t}-\psi \psi_{t t}^{*}+4 \lambda^{2}|\psi|^{2}-8 \lambda^{4},
\end{array}
$$

while

$$
\begin{aligned}
B_{p}(x, t)= & 2 \psi^{*}\left(3 \psi \psi_{t}^{*}-4 i \lambda^{3}\right)+2 i \lambda \psi_{t t}^{*} \\
& +\psi_{t t t}^{*}+4 i \lambda \psi^{*}|\psi|^{2}-4 \lambda^{2} \psi_{t}^{*}
\end{aligned}
$$

and

$$
\begin{aligned}
C_{p}(x, t)= & 2 \psi\left(3 \psi^{*} \psi_{t}+4 i \lambda^{3}\right)-2 i \lambda \psi_{t t} \\
& +\psi_{t t t}-4 i \lambda \psi|\psi|^{2}-4 \lambda^{2} \psi_{t} .
\end{aligned}
$$

Note that $B_{p}(x, t)=C_{p}(x, t)^{*}$ if $\lambda$ is real. Clearly, the linear functions $r$ and $s$ depend on the solution $\psi(x, t)$ that we use in the matrices.

Now the condition of compatibility (3) takes the form:

$$
\mathbf{B} \mathbf{L}-\mathbf{L} \mathbf{B}+\mathbf{B}_{t}-\mathbf{L}_{x}=0 .
$$

Expanding the l.h.s of (12) gives

$$
\left[\begin{array}{cc}
0 & F^{*}[\psi(x, y)] \\
-F[\psi(x, y)] & 0
\end{array}\right],
$$

where $F[\psi(x, y)]$ is defined in Eq.(2). Clearly, this is identically zero for any valid solution of Eq.(1), as $F[\psi(x, y)]=0$. Eq.(12) is correct for any complex $\lambda=a+i b$, and any real parameters $\alpha$ and $\gamma$. Thus, we have demonstrated the existence of the Lax pair and the spectral parameter $\lambda$ for Eq.(1).

As a next step, we use the above formalism to generate soliton solutions. This can be done using the Darboux transformations. We start with the seed solution $\psi_{0}=0$. Then the linear equations (3) take the form:

$$
\begin{aligned}
& \frac{1}{r} \frac{\partial r}{\partial t}=-\frac{1}{s} \frac{\partial s}{\partial t}=i \lambda \\
& \frac{1}{r} \frac{\partial r}{\partial x}=-\frac{1}{s} \frac{\partial s}{\partial x}=i \lambda^{2}\left(1-4 \alpha \lambda-8 \gamma \lambda^{2}\right),
\end{aligned}
$$

This set can be easily solved and we obtain:

$$
\begin{aligned}
& r=\exp \left[i \lambda t-i \lambda^{2} x\left(4 \alpha \lambda+8 \gamma \lambda^{2}-1\right)+i \phi\right], \\
& s=\exp \left[-i \lambda t+i \lambda^{2} x\left(4 \alpha \lambda+8 \gamma \lambda^{2}-1\right)-i \phi\right],
\end{aligned}
$$

where $\phi$ is a phase factor related to translations. Note that $s=1 / r$. These linear functions reduce to the functions found in the NLS case when $\alpha=\gamma=0$ [6].

Using these linear functions, we find the higher-order solution, $\psi_{1}$ without actually solving Eq.(1) through the algebraic expression

$$
\psi_{1}=\psi_{0}-\frac{2\left(\lambda^{*}-\lambda\right) s r^{*}}{|r|^{2}+|s|^{2}} .
$$

After simple substitution of $r$ and $s$, we obtain

$$
\psi_{1}=2 i b \operatorname{sech}[2 b(t+v x)] \exp [-i 2 \chi(x, t)],
$$

where

$$
v=2\left[a-16 \gamma a\left(a^{2}-b^{2}\right)-2 \alpha\left(3 a^{2}-b^{2}\right)\right]
$$

and

$$
\begin{aligned}
\chi(x, t) & =x\left[\left(a^{2}-b^{2}\right)-4 \alpha a\left(a^{2}-3 b^{2}\right)\right. \\
& \left.-8 \gamma\left(a^{4}-6 a^{2} b^{2}+b^{4}\right)\right]+a t+\phi .
\end{aligned}
$$

Clearly, (17) is one soliton solution of Eq.(1) with the amplitude $2 b$, velocity $v$ and the phase $\chi$.

Remarkably, the higher-order terms in Eq.(1) do not influence the soliton amplitude. Its value $2 b$ remains the same as for the soliton of the NLS. However, the soliton velocity $v$ in (18) becomes a complicated mix of soliton and equation parameters. Moreover, the directions of soliton propagation and the phase fronts propagation also differ. For the NLS case $(\alpha=\gamma=0), v=2 a, \chi=$ $\left(a^{2}-b^{2}\right) x+a t+\phi$ and the solution (17) reduces to the previously known result [6]. Then, the soliton propagates in the direction orthogonal to the phase fronts and its chirp is zero.

For simplicity, we take $\phi=0$. In order to obtain twosoliton solutions, we can use (15) to have two sets of first order linear functions $r_{1,1}=r\left(\lambda_{1}\right), s_{1,1}=s\left(\lambda_{1}\right)$ and $r_{1,2}=r\left(\lambda_{2}\right), s_{1,2}=s\left(\lambda_{2}\right)$ with $\lambda=\lambda_{1}$ and $\lambda=\lambda_{2}$ respectively. Thus,

$$
r_{1, j}=\exp \left[i \lambda_{j} t-i \lambda_{j}^{2} x\left(4 \alpha \lambda_{j}+8 \gamma \lambda_{j}^{2}-1\right)\right]
$$

while $s_{1, j}=1 / r_{1, j}$ where $j=1,2$. According to the Darboux transformations, higher order linear functions $r$ and $s$ can be calculated using just algebraic recurrent relations:

$$
\begin{aligned}
r_{n, j}= & \Delta\left[\left(\lambda_{n-1}^{*}-\lambda_{n-1}\right) s_{n-1,1}^{*} r_{n-1,1} s_{n-1, j+1}\right. \\
& +\left(\lambda_{j+n-1}-\lambda_{n-1}\right)\left|r_{n-1,1}\right|^{2} r_{n-1, j+1} \\
& \left.+\left(\lambda_{j+n-1}-\lambda_{n-1}^{*}\right)\left|s_{n-1,1}\right|^{2} r_{n-1, j+1}\right], \\
s_{n, j}= & \Delta\left[\left(\lambda_{n-1}^{*}-\lambda_{n-1}\right) s_{n-1,1} r_{n-1,1}^{*} r_{n-1, j+1}\right. \\
& +\left(\lambda_{j+n-1}-\lambda_{n-1}\right)\left|s_{n-1,1}\right|^{2} s_{n-1, j+1} \\
& \left.+\left(\lambda_{j+n-1}-\lambda_{n-1}^{*}\right)\left|r_{n-1,1}\right|^{2} s_{n-1, j+1}\right],
\end{aligned}
$$


instead of solving the linear equations (3). Here $\Delta=$ $\left(\left|r_{n-1,1}\right|^{2}+\left|s_{n-1,1}\right|^{2}\right)^{-1}$.

Now we obtain $\psi_{2}$ using linear functions (20) above in the recurrent expression for the solution of (1):

$$
\psi_{n}=\psi_{n-1}-\frac{2\left(\lambda_{n}^{*}-\lambda_{n}\right) s_{n, 1} r_{n, 1}^{*}}{\left|r_{n, 1}\right|^{2}+\left|s_{n, 1}\right|^{2}} .
$$

where the eigenvalues $\lambda_{j}$ are complex numbers: $\lambda_{j}=$ $a_{j}+i b_{j}$ with $j=1,2$.

To give a simple example of the two-soliton solution, we take the real and imaginary parts of the eigenvalues to be: $a_{1}=-a_{2}=1, b_{1}=b_{2}=1, \alpha=1 / 8, \gamma=1 / 16$. This choice provides equal amplitudes of the two solitons but different velocities. In this case the two soliton solution can be written in the form:

$$
\psi_{2}=2(1-i) \frac{N}{D} \exp [-2(1+i)(t+3 x)]
$$

where the numerator $N$ is

$$
N=e^{12 x}-i e^{4 i t+4(1+i) x}+e^{4(1+i) t+4(2+i) x}-i e^{4 t},
$$

while the denominator $D$ is

$$
D=\cos [4(t+x)]-\cosh [4(t-x)]-2 \cosh (8 x) .
$$

A plot of this solution is given in Fig.1. Clearly, this is a collision of two solitons with equal amplitudes. The collision is centred at the origin due to the choice of the phase $\phi=0$. The plot shows only the central, collision part of the solution.

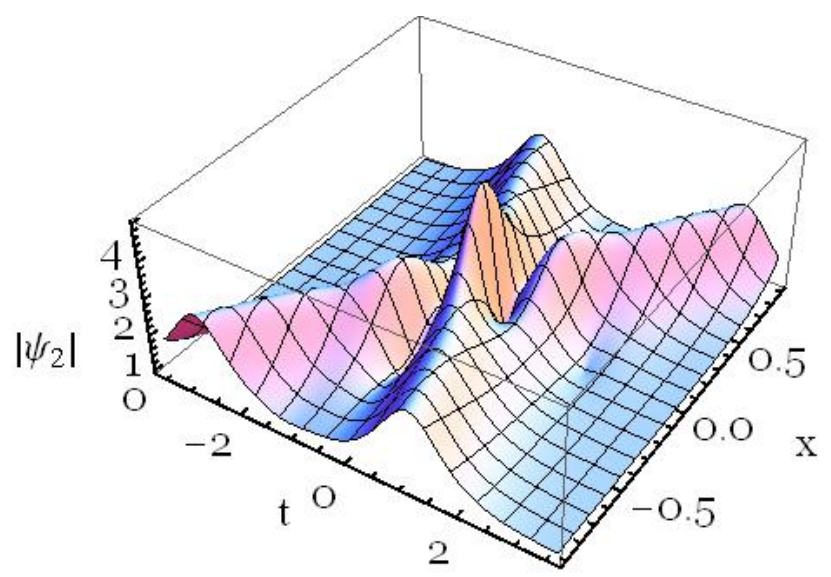

FIG. 1: Plot of the 2-soliton collision of eqn.(22). The two solitons have equal amplitudes, $b_{1}=b_{2}=1$ but opposite imaginary parts of the eigenvalues, $a_{1}=1, a_{2}=-1$. The equation parameters are: $\alpha=1 / 8$ and $\gamma=1 / 16$. The asymmetry in soliton velocities is caused by non-zero $\alpha$.

We now consider another special case of the two soliton solution. Parameters chosen for this case are $a_{1}=a_{2}=0$ and $b_{1}=1 / 2, b_{2}=3 / 2$. Equation parameters $\alpha$ and $\gamma$ are arbitrary. The solution is given by:

$$
\psi_{2}=4 i \frac{F_{b}}{D_{r}} \exp \left[i\left(\gamma+\frac{1}{2}\right) x\right]
$$

where

$$
F_{b}=3 e^{4 i(20 \gamma+1) x} \cosh (\alpha x+t)-\cosh [3(9 \alpha x+t)],
$$

and

$$
\begin{aligned}
D_{r} & =\cosh [4(7 \alpha x+t)]+4 \cosh [2(13 \alpha x+t)] \\
& -3 \cos [4(20 \gamma+1) x] .
\end{aligned}
$$

This is also a two-soliton solution with the amplitude of one soliton, $b_{2}$, being three times the amplitude of the other one, $b_{1}$. However, their velocities and positions are equal, and the two solitons propagate along the same line. Due to different propagation constants of the two solitons, there is periodic beating between them.

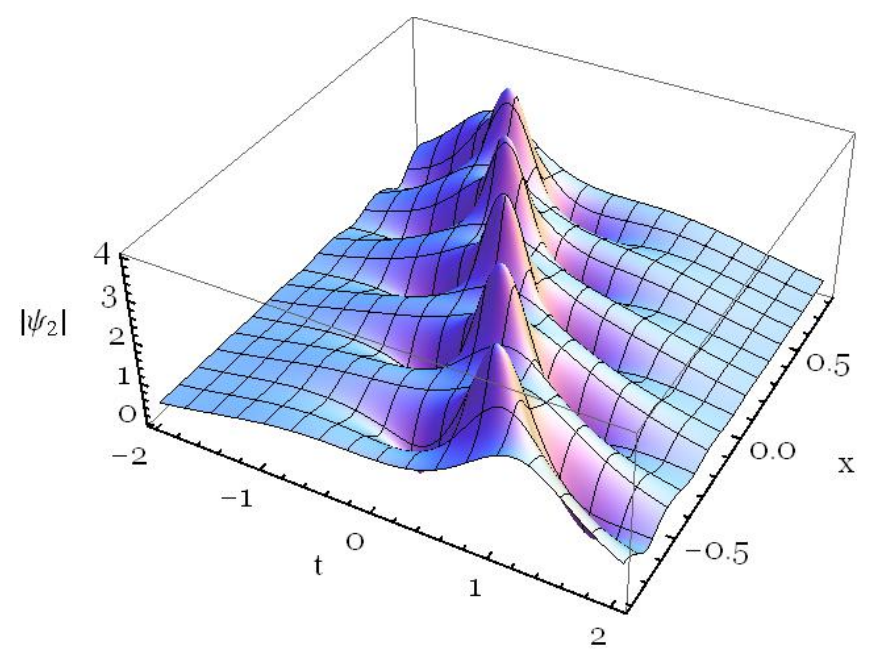

FIG. 2: Plot of the two-soliton breather solution of Eq.(23). Two solitons have equal velocities $a_{1}=a_{2}=0$, but different amplitudes $b_{1}=1 / 2, b_{2}=3 / 2$. The equation parameters are: $\alpha=1 / 8$ and $\gamma=1 / 5$. The maximum amplitude is 4 . The oblique propagation is caused by the nonzero $\alpha$.

An example of this solution with $\alpha=1 / 8$ and $\gamma=1 / 5$ is shown in Fig.2. The maximum amplitude $|q(0,0)|=4$, appears at the origin $x=0, t=0$ and the pulse compression recurs periodically. In the 'valleys' on either side of the origin, at $x \approx \pm 0.12$, the shape $|q|$ resembles $2 \operatorname{sech}(t)$. In the NLS case, it can be considered as the "higher order" soliton, with the valley having exactly the $2 \operatorname{sech}(t)$ profile. This breather is an analogue and generalisation of the Satsuma-Yajima solution of the NLS [17]. Although we have taken the real parts of the eigenvalues to be zero, the common velocity is nonzero due to the nonzero $\alpha$. The asymmetric part of the equation with $\alpha$ causes oblique propagation. This also happens for solutions of the Hirota equation [4, 7].

In the third example, we take both velocities and amplitudes of the two solitons to be equal, viz. $a_{1}=a_{2}=0$ and $b_{1}=b_{2}$. As we know [6], the solution becomes undefined when the two eigenvalues $\lambda_{i}$ coincide. However, the solution does exist [8]. This is so called degenerate case. In order to reveal the solution, the uncertainty has to be 
resolved using the L'Hopital rule. The solution found as a result of this procedure is:

$$
\psi_{2}=-8 b_{1} \frac{F}{D} \exp \left[2 i b_{1}^{2} x\left(8 b_{1}^{2} \gamma+1\right)\right]
$$

where the functions $F$ and $D$ are the mix of rational and hyperbolic expressions:

$$
\begin{aligned}
F= & \left(64 b_{1}^{4} \gamma x+4 b_{1}^{2} x-i\right) \cosh \left[2 b_{1}\left(4 \alpha b_{1}^{2} x+t\right)\right] \\
& +2 i b_{1}\left(12 \alpha b_{1}^{2} x+t\right) \sinh \left[2 b_{1}\left(4 \alpha b_{1}^{2} x+t\right)\right] \\
D= & 1+8 b_{1}^{2}\left[4 b_{1}^{2} x^{2}\left(36 \alpha^{2} b_{1}^{2}+\left(16 b_{1}^{2} \gamma+1\right)^{2}\right)\right. \\
& \left.+24 \alpha b_{1}^{2} t x+t^{2}\right]+\cosh \left[4 b_{1}\left(4 \alpha b_{1}^{2} x+t\right)\right] .
\end{aligned}
$$

The plot of this solution is given in Fig.3. This is an equivalent of the degenerate NLS solution (3.83) given in [6]. The maximum value of the central peak at the point of collision $\left|\psi_{2}(0,0)\right|=4 b_{1}$.

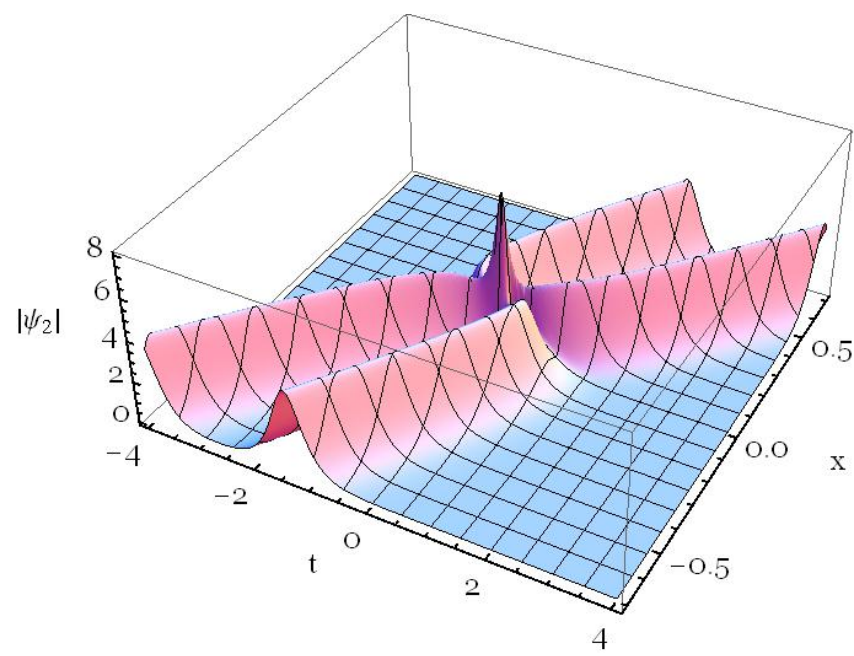

FIG. 3: Plot of degenerate two-solution solution (24). Two solitons have equal amplitudes $b_{1}=b_{2}=2$ and equal velocities $a_{1}=a_{2}=0$. The equation parameters are: $=-1 / 4$ and $\gamma=1 / 8$. The oblique propagation is caused by the nonzero $\alpha$.
The process of generation of higher-order solutions can be continued indefinitely. The third order solution requires three eigenvalues $\lambda_{i}$, and we apply the recursive relations three times. The graph presented in Fig.2.2 of [6] gives the algorithm of these calculations. The threesoliton solution, obtained this way is shown in Fig.4. We do not provide the explicit form of this solution as it is rather cumbersome.

In conclusion, we presented a new generalised NLS evolution equation with odd and even higher order terms with variable parameters to be integrable. To demonstrate integrability, we have given relevant higher order soliton solutions of this equation in explicit form.

$A A$ and NA acknowledge the support of the Australian Research Council (Discovery Project DP110102068) and

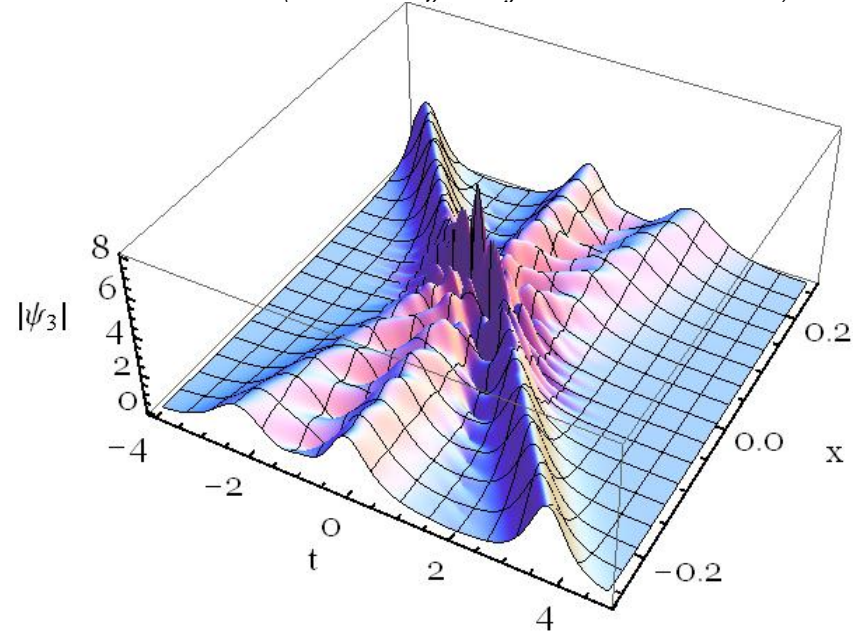

FIG. 4: Plot of the three-soliton collision (third order solution) obtained using the recursive formulae Eq.(20). The parameters are: $a_{1}=b_{1}=1, a_{2}=-3 / 2, b_{2}=4 / 3, a_{3}=3 / 2$, $b_{3}=5 / 3 ; \alpha=1 ; \gamma=1$.

also thank the Volkswagen Foundation for financial support.
[1] G. P. Agrawal, Nonlinear fiber optics (optics and photonics), 4 - th ed., (Academic press, 2010), Ch.4.

[2] A. Osborne, Nonlinear Ocean Waves and the Inverse Scattering Transform (Elsevier, 2010).

[3] V. E. Zakharov, A. B. Shabat, "Exact theory of two-dimensional self-focusing and one-dimensional selfmodulation of waves in nonlinear media", J. Experimental and Theoretical Physics, 34 (1): 6269 (1972).

[4] R. Hirota, Exact envelope-soliton solutions of a nonlinear wave equation, J. Math. Phys., 14, 805 (1973).

[5] M. Lakshmanan, K. Porsezian, M. Daniel, Effect of Discreteness on the Continuum Limit of the Heisenberg Spin
Chain,, Phys. Lett. A 133, 483 - 488 (1988).

[6] N. Akhmediev and A. Ankiewicz, Solitons, nonlinear pulses and beams, (Chapman and Hall, London, 1997). See sections. 2.7 and 3.16 .

[7] A. Ankiewicz, J. M. Soto-Crespo and N. Akhmediev, Rogue waves and rational solutions of the Hirota equation, Phys. Rev. E, 81, 046602 (2010).

[8] N. Akhmediev and A. Ankiewicz, Spatial soliton Xjunctions and couplers, Opt. Comm., 100, 186 - 192 (1993).

[9] K. Porsezian, M. Daniel, M. Lakshmanan, "On the integrability aspects of the one-dimensional classical con- 
tinuum isotropic Heisenberg spin chain", J. Math. Phys. 33, 1807 - 1816 (1992),

[10] K. Porsezian, "Completely integrable nonlinear Schrödinger type equations on moving space curves", Phys. Rev. E 55, 3785 - 3789 (1997).

[11] A. Ankiewicz, N. Devine and N. Akhmediev, "Are rogue waves robust against perturbations?", Physics Letters A, 373, 3997-4000 (2009).

[12] A. Ankiewicz, J. M. Soto-Crespo, M. A. Chowdhury and N. Akhmediev, 'Rogue waves in optical fibers in presence of third order dispersion, self-steepening and selffrequency shift', JOSA B 30, 87 - 94 (2013).

[13] N. Akhmediev, J. M. Dudley, D. R. Solli and S. K. Turitsyn, Recent progress in investigating optical rogue waves, J. Opt, 15, 060201 (2013).
[14] L. H. Wang, K. Porsezian and J. S. He, "Breather and rogue wave solutions of a generalized nonlinear Schrödinger equation", Phys. Rev. E 87, 053202 (2013).

[15] A. Ankiewicz, A. Chowdhury, N. Devine and N. Akhmediev, Rogue waves of the nonlinear Schrödinger equation with even symmetric perturbations, J. Opt., 15,064007 (2013).

[16] N. Akhmediev, A. Ankiewicz and J. M. Soto-Crespo, Rogue waves and rational solutions of the nonlinear Schrödinger equation, Phys. Rev. E 80, 026601 (2009).

[17] J. Satsuma, N. Yajima, "Initial value problems of onedimensional self-modulation of nonlinear waves in dispersive media", Prog. Theor. Phys. Suppl. 55, 284 (1974). 